\title{
Application of Primary/Secondary Circulating Tumor Cells for the Prediction of Biochemical Recurrence in Nonmetastatic Prostate Cancer Patients following Radical Prostatectomy or Radiotherapy: A Meta-Analysis
}

\author{
Liang Cao, ${ }^{1}$ Peng Hao, ${ }^{1}$ Dong Lin, ${ }^{2}$ Yangming Li, ${ }^{1}$ Tinghui Hu, ${ }^{3}$ Tao Cai, ${ }^{1}$ Shu Cui ${ }^{1},{ }^{1}$ \\ and Tao Wu iD ${ }^{1}$ \\ ${ }^{1}$ Department of Urology, Affiliated Hospital of North Sichuan Medical College, 1 Maoyuan South Road, Shunqing, Nanchong, \\ 637000 Sichuan, China \\ ${ }^{2}$ Department of Urology, Pengzhou People's Hospital, 255 South Third Ring Road, Chengdu, 611900 Sichuan, China \\ ${ }^{3}$ Department of Urology, Emeishan People's Hospital, 2 Santaishan Fifth Street, Emeishan, 614200 Sichuan, China \\ Correspondence should be addressed to Shu Cui; shucui2015@sina.com and Tao Wu; alhawking@163.com
}

Received 14 June 2021; Accepted 19 August 2021; Published 21 September 2021

Academic Editor: Robert Stoehr

Copyright (c) 2021 Liang Cao et al. This is an open access article distributed under the Creative Commons Attribution License, which permits unrestricted use, distribution, and reproduction in any medium, provided the original work is properly cited.

\begin{abstract}
Background. Circulating tumor cells (CTCs) have been regarded as an independent prognostic marker for metastatic castrationresistant prostate cancer (mCRPC). Its prognostic value, however, in nonmetastatic prostate cancer (NMPC) is still unclear. Purpose. To elucidate whether CTCs can predict the biochemical recurrence (BCR) in NMPC patients following radical prostatectomy (RP) or radiotherapy (RT). Methods. PubMed, Cochrane Database, and Embase and the references in relevant studies were systematically searched. Studies that investigated the correlation of CTCs and BCR in NMPC patients after RP or RT were identified and reviewed. Overall odds ratio (OR) of BCR in such patients with/without CTCs was pooled. We also calculated and pooled overall prevalence of BCR in such CTC-positive patients. Results. In total, 12 studies comprising 1917 participants were eligible for the meta-analysis and showed that the presence of secondary circulating tumor cells (SCTCs) is associated with a higher BCR rate of 59\% (95\% CI: 22\%-88\%) in patients with NMPC after RP or RT (OR = 6.12; 95\% CI: 2.22$16.85 ; P<0.001)$. However, regardless of the presence of primary circulating tumor cells (PCTCs), it has not been shown to be associated with higher BCR. Conclusions. Our research demonstrated that SCTC-positive patients are associated with higher BCR compared to SCTC-negative patients in NMPC. Therefore, it is recommended that NMPC patients undergo CTC surveillance intensively after RP or RT.
\end{abstract}

\section{Introduction}

Both radical prostatectomy $(\mathrm{RP})$ and radiotherapy $(\mathrm{RT})$ are standard therapies for treating nonmetastatic prostate cancer (NMPC) [1]. Despite being considered a localized disease at the beginning of any anticancer therapy, $15-30 \%$ and 10 $15 \%$ of patients will suffer biochemical recurrence (BCR) during the 5-year follow-up, respectively [2]. BCR is defined as detectable or rising prostate-specific antigen (PSA) value after surgery that is $\geq 0.2 \mathrm{ng} / \mathrm{ml}$ with a second confirmatory level of $\geq 0.2 \mathrm{ng} / \mathrm{ml}$ by the American Urological Association
(AUA) [3]. Similarly, it is also defined as a rise in PSA to $\geq 2 \mathrm{ng} / \mathrm{ml}$ above nadir PSA after external beam radiotherapy (EBRT) with or without hormonal treatment by the American Society for Radiation Oncology (ASTRO) and Radiation Therapy Oncology Group (RTOG) [4]. Meanwhile, some studies have reported that BCR is associated with a significantly increased risk of metastasis and $24-34 \%$ of patients with BCR will develop into metastasis $[5,6]$. Therefore, it is important to identify patients with high risk of treatment failure who should have benefited from early use of androgen deprivation therapy (ADT) or salvage radiotherapy (SRT) 
$[7,8]$. Clinical stage, Gleason score (GS), and PSA have all already been established as independent prognostic factors for prostate cancer patients [9]. Also, PSA is the first and only one serum marker for the diagnosis of prostate cancer with a positive predictive value of $47 \%$ approved by the U.S. Food and Drug Administration since 1986; it seems to neither predict response to therapy nor present tumor progression [10-12].

Many studies have reported that the BCR of prostate cancer was predicted in different ways with related markers, including methods based on RNA, gene expression, protein expression, and genomic alteration. Nevertheless, the stratified predicted effect of these methods is not satisfactory. Therefore, new related markers will be explored to fill this field, especially the detection of circulating tumor cells (CTCs) which may help to predict the response to anticancer therapy and has recently attracted great attention. Ashworth firstly found that tumor cells were released into the peripheral circulation of patients in 1869 [13], commonly known as CTCs now. A subpopulation of tumor cells, also called primary circulating tumor cells (PCTCs), disseminates first to the neurovascular structures and then into the circulation, most of which are eliminated by host defenses or destroyed by shear forces $[14,15]$. Though not all these cells are able to implant, PCTCs identify patients with possible clinically significant cancer. For example, those which express CD82, a tumor suppressor gene product linked to integration binding [16], are associated with upstaging and upgrading [17]. However, there may be a small part of PCTCs that will implant in distant tissues, survive, and in time proliferate. These cells will not be cleared by surgery and/or radiotherapy and may later be detected in the circulation which are called secondary circulating tumor cells (SCTCs) and thus represent minimal residual disease (MRD). CTC detection has been reported as a new noninvasive liquid-biopsy method applied in various solid tumors, such as breast cancer, pancreatic cancer, gastric cancer, and prostate cancer [18-21].

Recently, CTCs have been described as a reliable prognostic serum biomarker for metastatic castration-resistant prostate cancer (mCRPC) in many studies [22, 23]. However, some studies investigated the response of CTCs to RP or RT in NMPC showing different results [24, 25]. Consequently, we conducted a meta-analysis to investigate whether CTCs can predict the BCR in NMPC patients treated by RP or RT and to estimate the incidence rate of BCR in such CTC-positive patients.

\section{Methods}

The meta-analysis was conducted and reported according to the Preferred Reporting Items for Systematic Reviews and Meta-Analysis (PRISMA) statement [26], and the PRISMA checklist is detailed in the supplementary file (available here).

2.1. Data Sources and Search. A systematic literature search in PubMed, Cochrane Database, and Embase was conducted to identify relevant studies which investigated the correlation of CTCs and BCR in patients with NMPC after RP or
RT published through January 2021. The search strategy was based on the combination of the following keywords: prostate neoplasm, prostate cancer, prostatic neoplasm, prostatic cancer; prostatectomy, radical prostatectomy; radiotherapy, radiation therapy, radiation treatment, targeted radiotherapy; biochemical relapse, biochemical recurrence. The reference list was also checked to retrieve other papers related to our topic. Any disagreements between the two authors (LC and $\mathrm{PH}$ ) were settled by detailed discussion with a third investigator.

2.2. Study Selection. Two reviewers (LC and PH) independently reviewed the full texts of the potential eligible studies which met the following inclusion criteria: (1) the included subjects were NMPC patients treated by RP or RT and no evidence of BCR found when samples were collected; (2) the risk point estimate was reported as an odds ratio (OR) with the $95 \% \mathrm{CI}$, or the data were presented such that OR and 95\% CI could be calculated; (3) the samples used in these studies should be peripheral blood; (4) the language in which the articles are compiled should be English; and (5) conference abstracts, review articles, and meta-analysis will be excluded. Studies that did not meet the earlier criteria were excluded.

2.3. Data Extraction and Quality Assessment. Two reviewers independently extracted following data from all eligible studies: first author's name, year of publication, number of cases and controls, study design, time of blood collection (before or after any treatment), treatments used in each study, blood sample volume, and methods of enrichment and detection of CTCs. BCR was defined as patients who have undergone primary treatment with prostatectomy or radiation, with rise to $\geq 0.2$ from a prior undetectable level for prior prostatectomy or $>2 \mathrm{mg} / \mathrm{dl}$ rise from postnadir radiotherapy.

Because the risk of bias in nonrandomized studies of interventions (ROBINS-I) quality assessment tool is not suitable for the quality evaluation of observational cohort studies, the Newcastle-Ottawa Scale (NOS) is used to define the methodological quality of each study [27]. A score of 6-9 is defined as high methodological quality, whereas a score less than 6 is low quality. NOS quality scores are presented as part of descriptive summaries for each study and did not influence decisions to pool studies in the meta-analysis.

2.4. Data Synthesis and Meta-Analysis. The synthesis of the odds ratio $(\mathrm{OR})$ and the incidence rate of BCR was achieved by STATA ver14.0 (StataCorp, TX, USA) software and R ver4.0.4, respectively. Cochran's $Q$ statistic and $I^{2}$ statistic were used to assess the heterogeneity between all eligible studies. To explore studies which contribute to the heterogeneity of meta-analysis by omitting studies (leave one out at a time) from the meta-analysis, influence analysis would be performed if high heterogeneity $\left(I^{2}>50 \%\right)$ was found. Subgroup analysis was also used to explore the sources of heterogeneity. Similarly, the funnel chart was also used to assess publication bias. A 2 -sided $P<0.05$ was considered statistically significant for all statistical analysis. 


\section{Results}

3.1. Literature Search and Study Selection. The flowchart of literature screening and selection results based on the PRISMA statement is shown in Figure 1. 453 records were obtained by a detailed search of the electronic database according to our prespecified search strategy, and 37 were considered potentially suitable. A total of 12 studies with 1917 participants were considered eligible for inclusion in the final meta-analysis after full-text review $[16,17,24,25$, 28-35], and general characteristics of all included studies are shown in Table 1 . Of the 12 studies, 9 explored the relationship between CTCs and BCR in NMPC patients who would undergo RP or RT [17, 24, 25, 28, 29, 32-35], and 8 investigated such a correlation in NMPC patients who had undergone RP or RT without evidence of BCR [16, 28, 30-35]. Meanwhile, 5 studies simultaneously discussed the relationship between the existence of CTCs before and after any anticancer therapies and BCR [28, 32-35].

3.2. Association between PCTCs and BCR. We examined the relationship between PCTCs and BCR using data from 9 studies [17, 24, 25, 28, 29, 32-35]. There was no significant association between PCTCs and BCR $(\mathrm{OR}=2.07$; 95\% CI: $0.77-5.57 ; P=0.15 ; I^{2}=69 \%$ ) (Figure 2); the influence analysis showed the results were robust (Supplemental Figure 1). To explore the source of heterogeneity, we conducted a corresponding subgroup analysis; no significant relationship was found between PCTCs and BCR regardless of the treatment strategy (for RP $[17,24,25,28,29,32,33$, 35]: $\left.\mathrm{OR}=2.54 ; 95 \% \mathrm{CI}: 0.90-7.15 ; P=0.079 ; I^{2}=72.6 \%\right)$ (for RT $[25,34]$ : $\mathrm{OR}=0.48$; 95\% CI: 0.02-9.23; $P=0.625$; $I^{2}=36.9 \%$ ) (Supplemental Figure 2). There was also no significant association between PCTCs and BCR in studies using Cell Search System (CSS) to detect CTCs (OR $=0.87 ; 95 \%$ CI: $\left.0.40-1.89 ; P=0.725 ; I^{2}=0.0 \%\right)[24$, 32-34], whereas results from studies using other methods have shown a strong association between PCTCs and BCR $\left(\mathrm{OR}=4.56 ; 95 \%\right.$ CI: $\left.1.52-13.65 ; P=0.007 ; I^{2}=53.5 \%\right)$ [17, 25, 28, 29, 35] (Supplemental Figure 3).

3.3. Association between SCTCs and BCR. Eight studies focused on the relationship between SCTCs and BCR in NMPC patients treated by RP or RT [16, 28, 30-35], and a statistically significant association was found $(\mathrm{OR}=6.12$; 95\% CI: 2.22-16.85; $P<0.001 ; I^{2}=81.4 \%$ ) (Figure 3). As showed in Supplemental Figure 4, our results were stable even after the sensitivity analysis was performed. Then, the subgroup analysis was performed to make an attempt to explain heterogeneity. We found that the presence of SCTCs was strongly associated with BCR regardless of the treatment regimen used (for $\mathrm{RP}[16,28,31-33,35]$ : $\mathrm{OR}=5.84 ; 95 \% \mathrm{CI}$ : $1.36-25.08 ; P=0.018 ; I^{2}=84.9 \%$ ) (for RT $[30,34]$ : OR = 7.17; 95\% CI: 2.83-18.11; $P<0.001 ; I^{2}=10.7 \%$ ) (Supplemental Figure 5). Similarly, a statistically significant association was found between SCTCs and BCR in these studies that did not use CSS to detect CTCs $(\mathrm{OR}=9.33$; 95\% CI: 2.93-29.74; $\left.P<0.001 ; I^{2}=85.7 \%\right)[16,28,30,31,35]$. However, such a relationship has not been observed in these studies using CSS (OR $=1.96 ; 95 \% \mathrm{CI}: 0.58-6.58 ; P=0.278$; $I^{2}=0.0 \%$ ) [32-34] (Supplemental Figure 6).

3.4. BCR in CTC+ Patients. R software was used to integrate the incidence rate of BCR in primary or secondary CTC+ patients, and the incidence rate of BCR in primary and secondary CTC+ patients was 37\% (95\% CI: 9\%-66\%; $\left.I^{2}=99 \%\right)$ (Figure 4) and 59\% (95\% CI: 22\%-88\%; $I^{2}=66 \%$ ) (Figure 5), respectively. $65 \%$ of the BCR rate (95\% CI: $56 \%-73 \%$; $I^{2}=$ $0 \%$ ) (Supplemental Figure 7) was observed in these SCTC+ patients treated with RT $[30,34]$. We also found BCR rates of $47 \%$ (95\% CI: $19 \%-75 \%$; $\left.I^{2}=99 \%\right)[17,24,25,28,29$, 32, 33, 35] (Supplemental Figure 8) and 73\% (95\% CI: $40 \%-92 \% ; I^{2}=69 \%$ ) [16, 28, 31-33, 35] (Supplemental Figure 7) in patients who underwent RP with primary or secondary CTC+, respectively. Meanwhile, in studies with or without CSS, the cumulative incidence rate of BCR in PCTC+ patients was 26\% (95\% CI: 4\%-49\%; $\left.I^{2}=76 \%\right)$ [24, $32-34]$ and 43\% (95\% CI: 7\%-79\%; $\left.I^{2}=99 \%\right)$ [17, 25, 28, 29, 35], respectively (Supplemental Figure 9), while for SCTC+ patients, their incidence rate was 47\% (95\% CI: 30\%-64\%; $I^{2}$ $=0 \%)[32-34]$ and $71 \%$ (95\% CI: $\left.32 \%-93 \% ; I^{2}=81 \%\right)[16$, 28, 30, 31, 35], respectively (Supplemental Figure 10).

3.5. Publication Bias. Due to the limited number (below 10) of studies included in each meta-analysis, publication bias was not assessed.

\section{Discussion}

Meta-analysis of extractable data from 12 prospective studies demonstrated that the presence of SCTCs is associated with a higher BCR rate of 59\% (95\% CI: $22 \%-88 \%$ ) in NMPC patients after RP or RT. However, despite the $37 \%$ of BCR rate, we found no statistical association between PCTCs and BCR in such patients.

Owing to the fact that only 3 studies have investigated the relationship between CTCs and BCR in patients undergoing RT, the present meta-analysis shows that the presence of SCTCs in such patients associated with a BCR rate of $65 \%$ remained to be verified. The relationship with PCTCs cannot be estimated due to the absence of positive events. Given the low predicted rate now, CTC testing may not be appropriate to predict BCR in patients undergoing RT. Therefore, more prospective studies are needed to further confirm this result.

For NMPC patients treated with RP, our results demonstrated that SCTCs are associated with a higher BCR rate of $73 \%$. In other words, more than half of the patients that underwent RP will suffer from BCR once CTCs are detected. Chen et al. showed that CTCs in prostate cancer can be detected even without metastatic diseases and, therefore, that CTC monitoring has the potential to support early detection of disease progression in NMPC [36]. Meanwhile, a number of studies have demonstrated that early detection of cancer relapse or progression is associated with greater treatment options and better prognosis $[37,38]$. Given such a high incidence rate, early intervention of ADT and/or SRT in these patients is possible, resulting in significant long- 


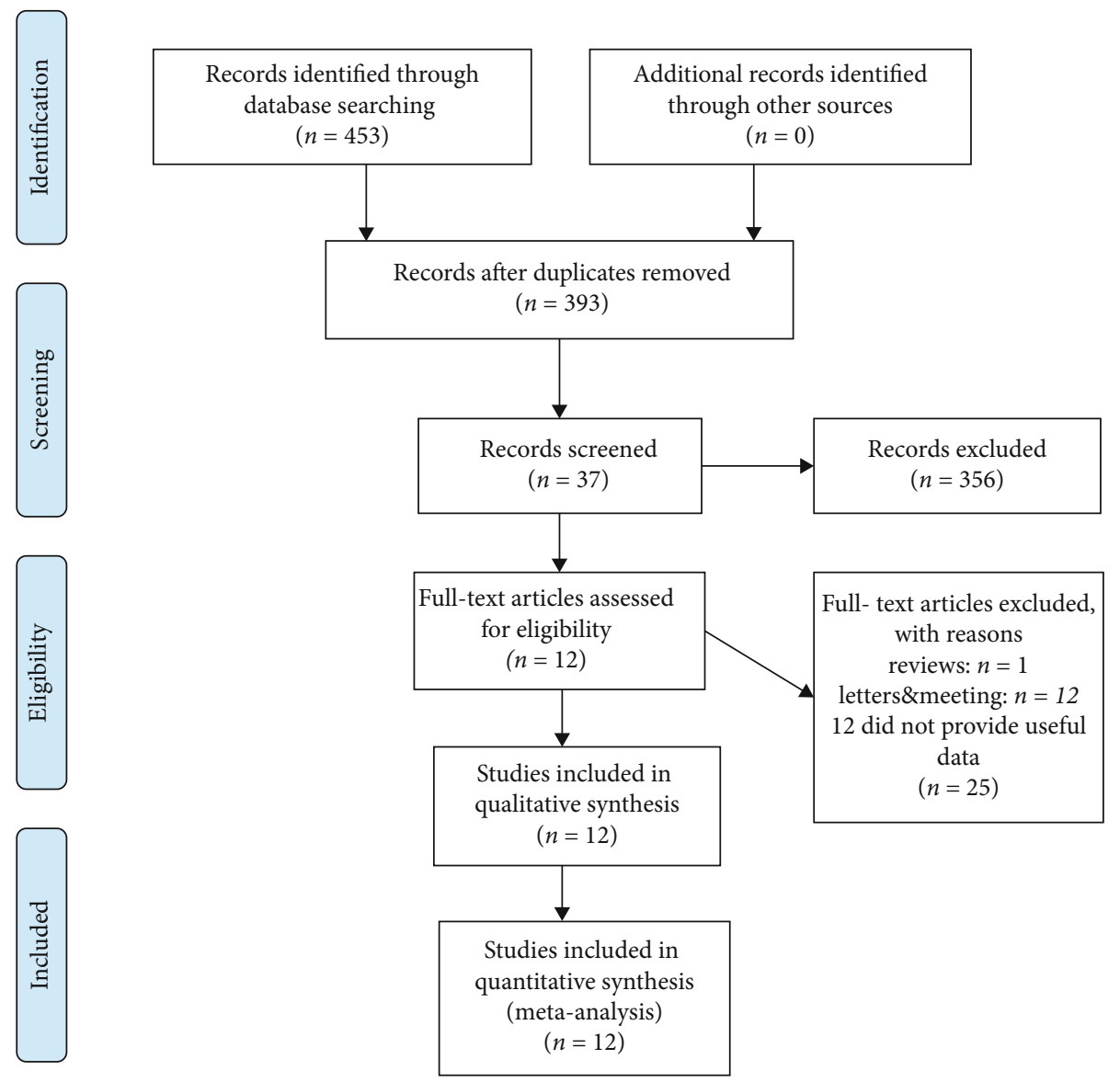

Figure 1: Flowchart for record selection process of the meta-analysis.

term prognostic benefits [8], and thus, excessive treatment could be avoided. Unfortunately, despite $37 \%$ of BCR rate, the association between PCTCs and BCR has not been found. In fact, two types of circulating prostate cells (CPCs) represent different clinical entities. SCTCs arising from a microfocus of micrometastatic diseases cannot be eliminated from the primary treatment and has great likelihood to lead to distant metastases compared with PCTCs that arise from the primary tumor, and not all of these PCTCs will survive or implant in distant tissues. Therefore, once the presence of SCTCs is detected, we should pay more attention to its indicative meaning and urge clinicians to intervene as early as possible.

One problem with CTC analysis is the variety of methods used to detect them, with varying degrees of specificity and sensitivity and the difference in their ability to detect malignant prostate cells rather than benign ones. Just as in benign colon diseases, CTCs which did not express P504S can be detected by CSS in men with benign prostate diseases [31, 39]. However, similar results and conclusions have been obtained in studies using different methods. Meyer et al. and Murray et al. both reported limited utility of PCTCs in predicting treatment outcome. Of the 12 included studies, 4 using CSS to detect CTCs demonstrated that no association between the presence of primary or secondary CTCs and BCR in NMPC patients after RP or
RT was found. Meanwhile, a relatively poor BCR prediction rate was found in studies using the CSS method for PCTC+ $(26 \%)$ or SCTCs+ $(47 \%)$ patients, respectively. Actually, the number of CTCs detected by the CSS is underestimated in NMPC, which may be due to the fragmentation of traditional CTCs and disability to identify CTCs lacking epithelial characteristics [40]. At the same time, some studies have demonstrated that relying on epithelial cell adhesion molecule- (EPCAM-) based enrichment methods alone cannot completely detect all CTCs [41]. This may explain why the results of the data synthesis of studies using CSS did not show the correlation between primary/secondary CTCs and BCR. Of course, apart from the CSS, there are multiple methods to detect CTCs, including real-time polymerase chain reaction, cell size-based separation, or immunomagnetic beads conjugated with anti-EpCAM antibodies. The results obtained from studies that did not use the CSS show a great connection between primary/secondary CTCs and BCR in NMPC. Therefore, based on the current evidence, the conclusions drawn from studies using CSS need to be interpreted cautiously.

Up to now, CTCs have been regarded as an independent prognostic marker for $\mathrm{mCRPC}$, but their prognostic value in NMPC is still unclear. This is the first meta-analysis to investigate whether the presence of CTCs is associated with a higher BCR rate in NMPC following RP or RT. In previous 


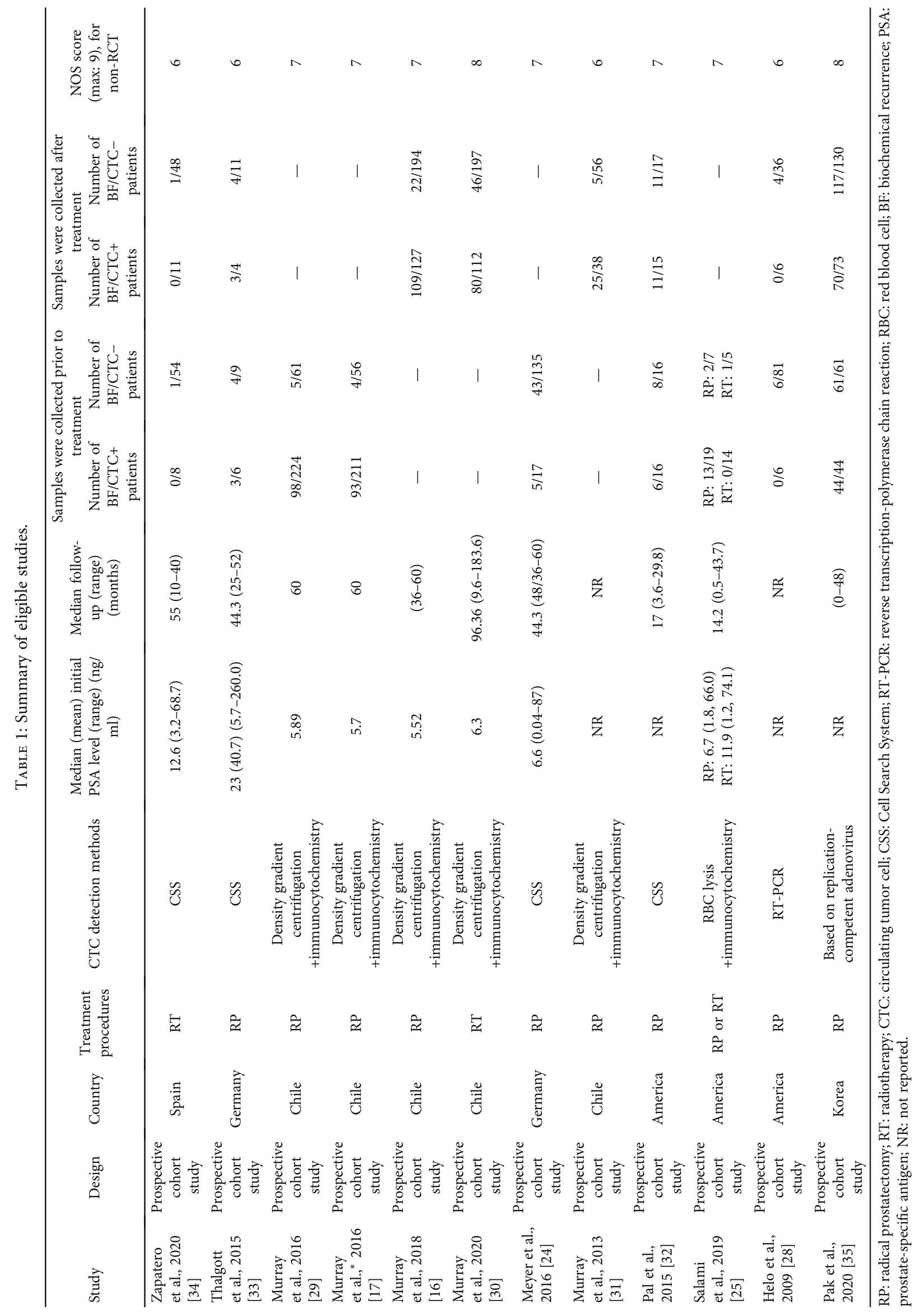


Study

ID
Events, Events, \%

OR (95\% CI) Study Control Weight

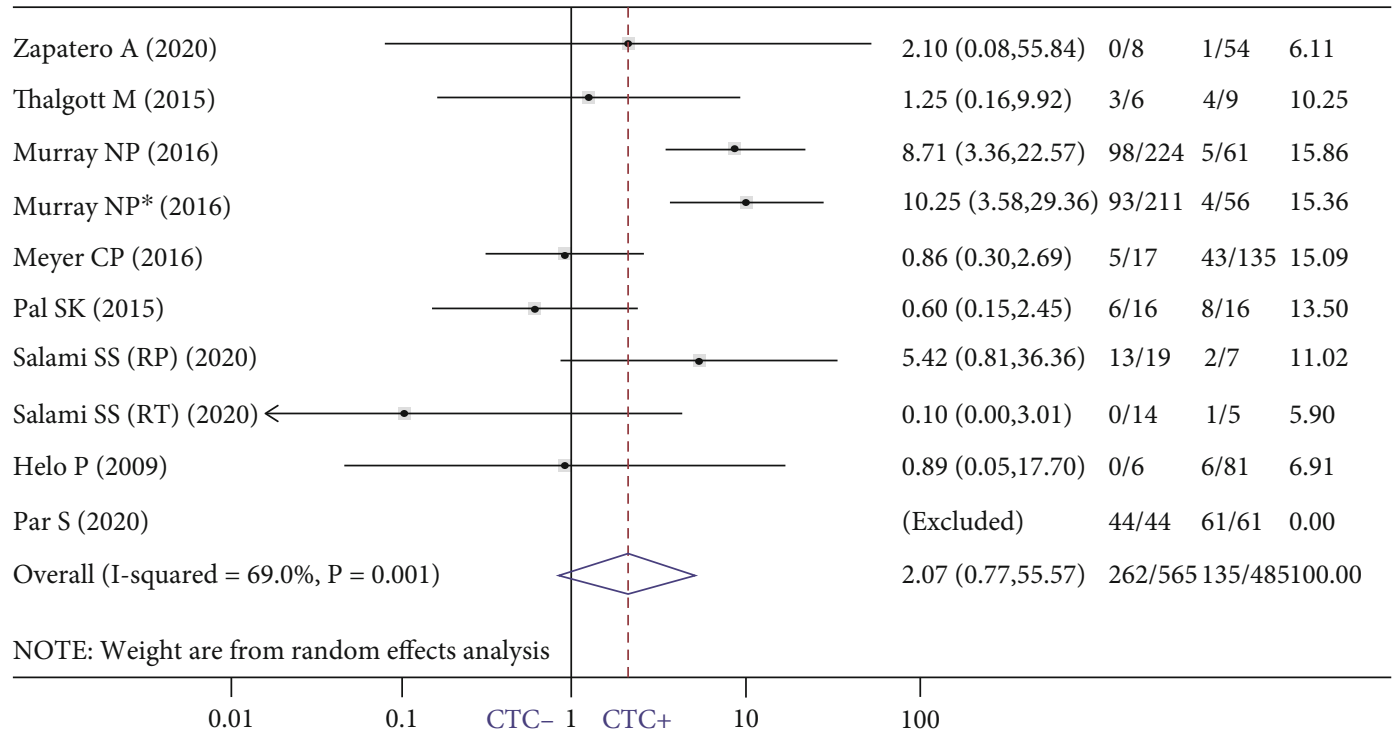

FIGURE 2: Pooled estimate of the association of primary circulating tumor cell-positive with biochemical recurrence.

Study

ID
Events Events \%

OR $(95 \%$ CI $) \quad$ Study Control Weight

\begin{tabular}{|c|c|c|c|c|c|}
\hline Zapatero A (2020) & $\rightarrow$ & $1.38(0.05,36.04)$ & $0 / 11$ & $1 / 48$ & 6.37 \\
\hline Thalgott M (2015) & & $5.25(0.40,68.95)$ & $3 / 4$ & $4 / 11$ & 8.49 \\
\hline Murray NP (2018) & & $\longrightarrow \quad 47.34(24.29,92.29)$ & $109 / 127$ & $22 / 194$ & 17.39 \\
\hline Murray NP (2020) & & $8.21(4.85,13.89)$ & $80 / 112$ & $46 / 197$ & 17.91 \\
\hline Murray NP (2013) & & $19.62(6.29,61.15)$ & $25 / 38$ & $5 / 56$ & 15.21 \\
\hline Fal SK (2015) & - & $1.50(0.33,11.62)$ & $11 / 15$ & $11 / 17$ & 13.24 \\
\hline Helo P (2009) & 7 & $0.56(0.03,11.62)$ & $0 / 6$ & $4 / 36$ & 6.98 \\
\hline Fak S $2020(2020)$ & 1 & $2.59(0.71,9.42)$ & $70 / 73$ & $117 / 130$ & 14.42 \\
\hline O-verall $(I$-squared $=81.4 \%, p=0.000)$ & & $6.12(2.22,16.85)$ & $298 / 386$ & $210 / 689$ & 100.0 \\
\hline Note: W-eights are from random effects analysi & & & & & \\
\hline $\begin{array}{c}1 \\
0.01\end{array}$ & CTC + & $\begin{array}{c}1 \\
100\end{array}$ & & & \\
\hline
\end{tabular}

FIgURe 3: Pooled estimate of the association of secondary circulating tumor cell-positive with biochemical recurrence.

reports, a CTC detection rate of $5-27 \%$ was found in NMPC [42-44], and one thing that can be observed is that the detection rate of CTCs in NMPC is relatively low. Therefore, CTC+ would be clinically meaningful in predicting the longterm prognosis of these items, although our conclusions demonstrate that only the presence of SCTCs is associated with a higher BCR rate of $59 \%$ independent of the method used to detect them. Meanwhile, compared with the Walz nomogram, PCTCs did not predict BCR, whereas SCTCs did [17, 29]. In the 2019 European Urological Association Guideline, it is recommended that patients with BCR after $\mathrm{RP}$ undergo positron tomography computed tomography (PET-CT) examination, and for patients with BCR undergoing RT, multiparameter magnetic resonance imaging (MPMRI) is recommended to localize abnormal areas and guide biopsies; also perform PET-CT with PSMA or fluciclovine or choline screening in these patients who are suitable for SRT [45]. Admittedly, the presence of CTCs in NMPC patients 


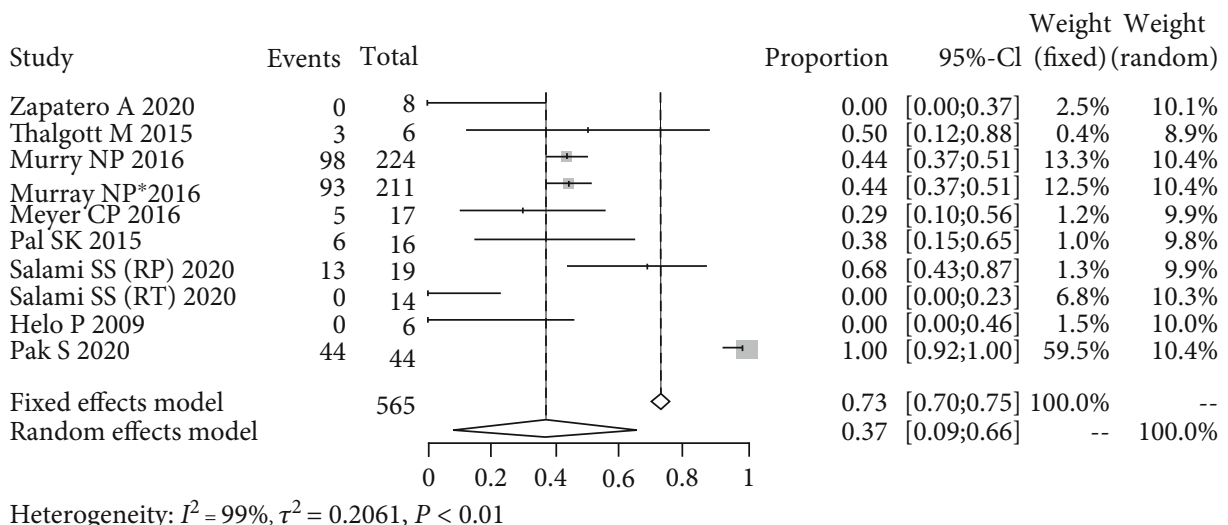

FIgURe 4: Pooled estimate of the incidence of biochemical recurrence in patients with primary circulating tumor cells.

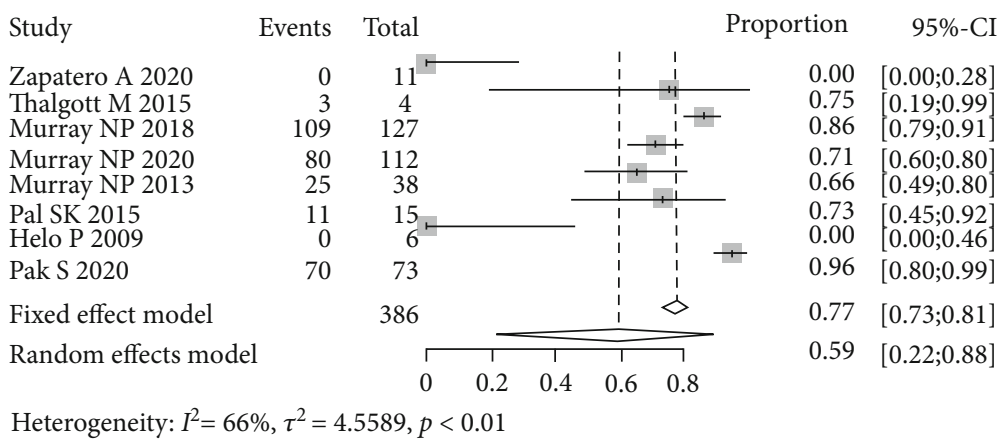

FIGURE 5: Pooled estimate of the incidence of biochemical recurrence in patients with secondary circulating tumor cells.

after RP or RT did not indicate whether tumor micrometastasis has occurred, but it can provide sufficient intervention intervals to reduce micrometastasis. Our results revealed that more than half of these SCTC+ patients would suffer from BCR. If these SCTC+ patients were provided routinely with the above screening only performed after BCR, will it detect early metastases while avoiding the problem of excessive examination? This is a question worth exploring and more research is needed to confirm it. Although the timing and treatment modality for PSA-only recurrences after RP or RT remain controversial based on the limited evidence, early SRT provides the possibility of cure for patients with an increasing PSA after RP. Boorjian et al. reported a $75 \%$ reduced risk of systemic progression with SRT, when comparing 856 SRT patients with 1801 non-SRT patients [46]. A retrospective analysis of 635 patients who were followed up after RP and experienced BCR and/or local recurrence and either received no salvage treatment $(n=397)$ or salvage RT alone $(n=160)$ within two years of BCR showed that salvage RT was associated with a threefold increase in prostate cancer-specific survival relative to those who received no salvage treatment [47]. Therefore, SRT is worth considering for NMPC patients following RP or RT with BCR, and it is worthy of cautious discussion to perform SRT especially for such SCTC+ patients. Thus, further studies, especially prospective studies, are required to advance knowledge in this field.

Several limitations of our meta-analysis are worth discussing. Although all eligible studies are prospective, selection bias cannot be avoided due to the fact that 5 of the eligible studies were done by the same team. At the same time, an article with a small sample size is included in this article, which may amplify the final conclusion.

\section{Conclusions}

Our research demonstrated that SCTC-positive patients are associated with higher BCR compared to SCTC-negative patients in NMPC. Therefore, it is recommended that NMPC patients undergo CTC surveillance intensively after RP or RT. Further studies, especially prospective studies, are required to better elucidate these relationships and to advance knowledge in this field.

\section{Data Availability}

The data used to support the findings of this study are included within the article. 


\section{Disclosure}

The patients or the public were not involved in the design, or conduct, or reporting, or dissemination plans of our research.

\section{Conflicts of Interest}

The authors declare that there is no conflict of interest regarding the publication of this paper.

\section{Authors' Contributions}

Project development and public funding were done by SC and TW. Data collection was done by DL, THH, and YML. Data analysis was done by LC, PH, and TC. Manuscript writing/editing was done by all the authors. Final approval of the manuscript was done by all the authors. Liang Cao and Peng Hao contributed equally to this work.

\section{Acknowledgments}

This work was supported by the City of Nanchong Strategic Cooperation with Local Universities Foundation of Technology (NSMC20170421, NSMC20170111, 18SXHZ0581, and 18SXHZ0128) and by Application and Basic Research Program of Sichuan Science and Technology Department (2020YJ0185).

\section{Supplementary Materials}

Supplemental Figure 1: influence analysis of meta-analysis of relationship between primary circulating tumor cell-positive and biochemical recurrence. Supplemental Figure 2: pooled estimate of subgroup analysis in the association of primary circulating tumor cell-positive with biochemical recurrence: radical prostatectomy vs. radiotherapy. Supplemental Figure 3: pooled estimate of subgroup analysis in the association of primary circulating tumor cell-positive with biochemical recurrence: Cell Search System vs. other tools. Supplemental Figure 4: influence analysis of meta-analysis of relationship between secondary circulating tumor cell-positive with biochemical recurrence. Supplemental Figure 5: pooled estimate of subgroup analysis in the association of secondary circulating tumor cell-positive with biochemical recurrence: radical prostatectomy vs. radiotherapy. Supplemental Figure 6: pooled estimate of subgroup analysis in the association of secondary circulating tumor cell-positive with biochemical recurrence: Cell Search System vs. other tools. Supplemental Figure 7: pooled estimate of subgroup analysis in the incidence of biochemical recurrence in patients with secondary circulating tumor cells: radical prostatectomy vs. radiotherapy. Supplemental Figure 8: pooled estimate of subgroup analysis in the incidence of biochemical recurrence in patients with primary circulating tumor cells: radical prostatectomy vs. radiotherapy. Supplemental Figure 9: pooled estimate of subgroup analysis in the incidence of biochemical recurrence in patients with primary circulating tumor cells: Cell Search System vs. other tools. Supplemental Figure 10: pooled estimate of subgroup analysis in the incidence of biochemical recurrence in patients with secondary circulat- ing tumor cells: Cell Search System vs. other tools. We have provided a list of PRISMA-P; see file PRISMA-PChecklist.docx for details. (Supplementary Materials)

\section{References}

[1] M. Han, A. W. Partin, M. Zahurak, S. Piantadosi, J. I. Epstein, and P. C. Walsh, "Biochemical (prostate specific antigen) recurrence probability following radical prostatectomy for clinically localized prostate cancer," Journal of Urology, vol. 169, no. 2, pp. 517-523, 2003.

[2] A. D. Vassil, E. S. Murphy, C. A. Reddy et al., "Five year biochemical recurrence free survival for intermediate risk prostate cancer after radical prostatectomy, external beam radiation therapy or permanent seed implantation," Brachytherapy, vol. 76, no. 5, pp. 1251-1257, 2010.

[3] I. M. Thompson, R. K. Valicenti, P. Albertsen et al., “Adjuvant and salvage radiotherapy after prostatectomy: AUA/ASTRO guideline," The Journal of Urology, vol. 190, no. 2, pp. 441449, 2013.

[4] M. Roach 3rd, G. Hanks, H. Thames Jr. et al., "Defining biochemical failure following radiotherapy with or without hormonal therapy in men with clinically localized prostate cancer: recommendations of the RTOG-ASTRO Phoenix Consensus Conference," International Journal of Radiation Oncology • Biology • Physics, vol. 65, no. 4, pp. 965-974, 2006.

[5] S. A. Boorjian, R. H. Thompson, M. K. Tollefson et al., "LongTerm Risk of Clinical Progression After Biochemical Recurrence Following Radical Prostatectomy: The Impact of Time from Surgery to Recurrence," European Urology, vol. 59, no. 6, pp. 893-899, 2011.

[6] C. R. Pound, A. W. Partin, M. A. Eisenberger, D. W. Chan, J. D. Pearson, and P. C. Walsh, "Natural history of progression after PSA elevation following radical prostatectomy," JAMA, vol. 281, no. 17, pp. 1591-1597, 1999.

[7] E. Messing, J. Manola, J. Yao et al., "Immediate versus deferred androgen deprivation treatment in patients with node-positive prostate cancer after radical prostatectomy and pelvic lymphadenectomy," The Lancet Oncology, vol. 7, no. 6, pp. 472479, 2006.

[8] I. Thompson, C. Tangen, J. Paradelo et al., "Adjuvant radiotherapy for pathological T3N0M0 prostate cancer significantly reduces risk of metastases and improves survival: long-term followup of a randomized clinical trial," The Journal of Urology, vol. 181, no. 3, pp. 956-962, 2009.

[9] P. J. Chuba, J. Moughan, J. D. Forman, J. Owen, and G. Hanks, "The 1989 patterns of care study for prostate cancer: five-year outcomes," International Journal of Radiation Oncology • Biology • Physics, vol. 50, no. 2, pp. 325-334, 2001.

[10] M. K. Brawer, M. C. Benson, D. G. Bostwick et al., "Prostatespecific antigen and other serum markers: current concepts from the World Health Organization Second International Consultation on Prostate Cancer," Seminars in Urologic Oncology, vol. 17, no. 4, pp. 206-221, 1999.

[11] W. R. Farwell, J. A. Linder, and A. K. Jha, "Trends in prostatespecific antigen testing from 1995 through 2004," Archives of Internal Medicine, vol. 167, no. 22, pp. 2497-2502, 2007.

[12] J. C. Gonzales, L. M. Fink, O. B. Goodman Jr., J. T. Symanowski, N. J. Vogelzang, and D. C. Ward, "Comparison of circulating microRNA 141 to circulating tumor cells, lactate dehydrogenase, and prostate-specific antigen for determining treatment response in patients with 
metastatic prostate cancer," Clinical Genitourinary Cancer, vol. 9, no. 1, pp. 39-45, 2011.

[13] T. R. Ashworth, "A case of cancer in which cells similar to those in the tumours were seen in the blood after death," The Australasian Medical Journal, vol. 14, p. 146, 1869.

[14] I. J. Fidler, "Metastasis: quantitative analysis of distribution and fate of tumor emboli labeled with 125 I-5-iodo-2'-deoxyuridine," Journal of the National Cancer Institute, vol. 45, no. 4, pp. 773-782, 1970.

[15] J. G. Moreno, C. M. Croce, R. Fischer et al., "Detection of hematogenous micrometastasis in patients with prostate cancer," Cancer Research, vol. 52, no. 21, pp. 6110-6112, 1992.

[16] N. P. Murray, S. Aedo, C. Fuentealba, E. Reyes, and O. Jacob, "Combining the prostate cancer risk index (PRIX) with the presence of secondary circulating prostate cells to predict the risk of biochemical failure after radical prostatectomy for prostate cancer," Asian Pacific Journal of Cancer Prevention, vol. 19, no. 12, pp. 3375-3381, 2018.

[17] N. P. Murray, E. Reyes, N. Orellana, C. Fuentealba, S. Orellana, and O. Jacob, "Does the presence of primary circulating prostate cells imply the presence of agressive prostate cancer with early biochemical failure: a comparison with the Walz nomogram," Asian Pacific Journal of Cancer Prevention, vol. 17, no. 7, pp. 3089-3093, 2016.

[18] L. Zhang, S. Riethdorf, G. Wu et al., "Meta-analysis of the prognostic value of circulating tumor cells in breast cancer," Clinical Cancer Research, vol. 18, no. 20, pp. 5701-5710, 2012.

[19] L. Han, W. Chen, and Q. Zhao, "Prognostic value of circulating tumor cells in patients with pancreatic cancer: a meta-analysis," Tumor Biology, vol. 35, no. 3, pp. 2473-2480, 2014.

[20] X. Ma, Z. Xiao, X. Li et al., "Prognostic role of circulating tumor cells and disseminated tumor cells in patients with prostate cancer: a systematic review and meta-analysis," Tumour Biology, vol. 35, no. 6, pp. 5551-5560, 2014.

[21] S. Wang, G. Zheng, B. Cheng et al., "Circulating tumor cells (CTCs) detected by RT-PCR and its prognostic role in gastric cancer: a meta-analysis of published literature," PLoS One, vol. 9, no. 6, article e99259, 2014.

[22] G. Heller, R. McCormack, T. Kheoh et al., "Circulating tumor cell number as a response measure of prolonged survival for metastatic castration-resistant prostate cancer: a comparison with prostate-specific antigen across five randomized phase III clinical trials," Journal of Clinical Oncology, vol. 36, no. 6, pp. 572-580, 2018.

[23] H. I. Scher, X. Jia, J. S. de Bono et al., "Circulating tumour cells as prognostic markers in progressive, castration- resistant prostate cancer: a reanalysis of IMMC38 trial data," The Lancet Oncology, vol. 10, no. 3, pp. 233-239, 2009.

[24] C. P. Meyer, K. Pantel, P. Tennstedt et al., "Limited prognostic value of preoperative circulating tumor cells for early biochemical recurrence in patients with localized prostate cancer," Urologic Oncology, vol. 34, no. 5, pp. 235.e11-235.e16, 2016.

[25] S. S. Salami, U. Singhal, D. E. Spratt et al., "Circulating tumor cells as a predictor of treatment response in clinically localized prostate cancer," JCO Precision Oncology, vol. 3, no. 3, pp. 1-9, 2019.

[26] D. Moher, A. Liberati, J. Tetzlaff, D. G. Altman, and for the PRISMA Group, "Preferred reporting items for systematic reviews and meta-analyses: the PRISMA statement," BMJ, vol. 339, no. jul21 1, p. b2535, 2009.
[27] G. Wells, B. Shea, D. O’Connell et al., “The Newcastle-Ottawa scale (NOS) for assessing the quality of non-randomised studies in meta-analyses," 2012, http://www.Ohri.ca/Programs/ Clinical_epidemiology/Oxford.Asp.

[28] P. Helo, A. M. Cronin, D. C. Danila et al., "Circulating prostate tumor cells detected by reverse transcription-PCR in men with localized or castration-refractory prostate cancer: concordance with CellSearch assay and association with bone metastases and with survival," Clinical Chemistry, vol. 55, no. 4, pp. 765-773, 2009.

[29] N. P. Murray, S. Aedo, C. Fuentealba et al., "Limited improvement of incorporating primary circulating prostate cells with the CAPRA score to predict biochemical failure-free outcome of radical prostatectomy for prostate cancer," Urologic Oncology, vol. 34, no. 10, pp. 430.e17-430.e25, 2016.

[30] N. P. Murray, S. Aedo, C. Fuentealba et al., "The CAPRA score versus sub-types of minimal residual disease to predict biochemical failure after external beam radiotherapy," ecancermedicalscience, vol. 14, 2020.

[31] N. P. Murray, E. Reyes, N. Orellana et al., "Secondary circulating prostate cells predict biochemical failure in prostate cancer patients after radical prostatectomy and without evidence of disease," ScientificWorldJournal, vol. 2013, article 762064, 7 pages, 2013.

[32] S. K. Pal, M. He, T. Wilson et al., "Detection and phenotyping of circulating tumor cells in high-risk localized prostate cancer," Clinical Genitourinary Cancer, vol. 13, no. 2, pp. 130136, 2015.

[33] M. Thalgott, B. Rack, T. Horn et al., "Detection of circulating tumor cells in locally advanced high-risk prostate cancer during neoadjuvant chemotherapy and radical prostatectomy," Anticancer Research, vol. 35, no. 10, pp. 5679-5685, 2015.

[34] A. Zapatero, A. Gómez-Caamaño, M. Cabeza Rodriguez et al., "Detection and dynamics of circulating tumor cells in patients with high-risk prostate cancer treated with radiotherapy and hormones: a prospective phase II study," Radiation Oncology, vol. 15, no. 1, p. 137, 2020.

[35] S. Pak, Y. S. Suh, D. E. Lee et al., "Association between postoperative detection of circulating tumor cells and recurrence in patients with prostate cancer," The Journal of Urology, vol. 203, no. 6, pp. 1128-1134, 2020.

[36] S. Chen, G. Tauber, T. Langsenlehner et al., "In vivo detection of circulating tumor cells in high-risk non-metastatic prostate cancer patients undergoing radiotherapy," Cancers, vol. 11, no. 7, p. 933, 2019.

[37] J. Tol, M. Koopman, M. Miller et al., "Circulating tumour cells early predict progression-free and overall survival in advanced colorectal cancer patients treated with chemotherapy and targeted agents," Annals of Oncology, vol. 21, no. 5, pp. 10061012, 2010.

[38] D. Hayes, M. Cristofanilli, G. Budd et al., "Circulating tumor cells at each follow-up time point during therapy of metastatic breast cancer patients predict progression-free and overall survival," Clinical Cancer Research, vol. 12, no. 14, pp. 4218-4224, 2006.

[39] K. Pantel, E. Denève, D. Nocca et al., "Circulating epithelial cells in patients with benign colon diseases," Clinical Chemistry, vol. 58, no. 5, pp. 936-940, 2012.

[40] J. Davis, H. Nakanishi, V. Kumar et al., "Circulating tumor cells in peripheral blood samples from patients with increased serum prostate specific antigen: initial results in early prostate 
cancer," The Journal of Urology, vol. 179, no. 6, pp. 2187-2191, 2008, discussion 2191.

[41] H. Schneck, B. Gierke, F. Uppenkamp et al., "EpCAM-independent enrichment of circulating tumor cells in metastatic breast cancer," PLoS One, vol. 10, no. 12, article e0144535, 2015.

[42] L. Resel Folkersma, L. San José Manso, I. Galante Romo, J. Moreno Sierra, and C. Olivier Gómez, "Prognostic significance of circulating tumor cell count in patients with metastatic hormone-sensitive prostate cancer," Urology, vol. 80, no. 6, pp. 1328-1332, 2012.

[43] M. Thalgott, B. Rack, T. Maurer et al., "Detection of circulating tumor cells in different stages of prostate cancer," Journal of Cancer Research and Clinical Oncology, vol. 139, no. 5, pp. 755-763, 2013.

[44] J. Loh, L. Jovanovic, M. Lehman et al., "Circulating tumor cell detection in high-risk non-metastatic prostate cancer," Journal of Cancer Research and Clinical Oncology, vol. 140, no. 12, pp. 2157-2162, 2014.

[45] T. B. L. Lam, S. MacLennan, P. M. Willemse et al., "EAUEANM-ESTRO-ESUR-SIOG prostate cancer guideline panel consensus statements for deferred treatment with curative intent for localised prostate cancer from an international collaborative study (DETECTIVE study)," European Urology, vol. 76, no. 6, pp. 790-813, 2019.

[46] S. A. Boorjian, R. J. Karnes, P. L. Crispen, L. J. Rangel, E. J. Bergstralh, and M. L. Blute, "Radiation therapy after radical prostatectomy: impact on metastasis and survival," The Journal of Urology, vol. 182, no. 6, pp. 2708-2715, 2009.

[47] B. J. Trock, M. Han, S. J. Freedland et al., "Prostate cancerspecific survival following salvage radiotherapy vs observation in men with biochemical recurrence after radical prostatectomy," JAMA, vol. 299, no. 23, pp. 2760-2769, 2008. 